\title{
CAPITULO 43
}

\section{EFICÁCIA DOS EXERCÍCIOS NA DOR EM PACIENTES COM OSTEOARTRITE DE JOELHO: UMA REVISÃO SISTEMÁTICA COM METANÁLISE}

\section{DOI 10.4322/978-65-995353-2-1.c43}

Lílian Maria Magalhães Costa de Oliveira ${ }^{1}$, Maria Yasmim da Conceição Chagas ${ }^{2}$, Monaliza de Sousa Moura ${ }^{3}$, Verônica Letícia Magalhães da Silva ${ }^{4}$, Bruna Steffany Aquino de Oliveira ${ }^{5}$, Mayara Monteiro Andrade ${ }^{6}$, Mariana Antonia de Carvalho Ferreira $^{7}$, Ravena Mayra Sousa Braga ${ }^{8}$, Jorgyanne Gonzalez Costa ${ }^{9}$, Laiana Sepúlveda

\author{
de Andrade Mesquita ${ }^{10}$ \\ ${ }^{1}$ Universidade Estadual do Piauí, (liliancostaa_@hotmail.com) \\ ${ }^{2}$ Universidade Estadual do Piauí, (yasmimchagaslp@gmail.com) \\ ${ }^{3}$ Universidade Estadual do Piauí, (monalizasmoura@outlook.com) \\ ${ }^{4}$ Universidade Estadual do Piauí, (magalhaesveronicals@gmail.com) \\ ${ }^{5}$ Universidade Estadual do Piauí, (brunasteffany2980@gmail.com) \\ ${ }^{6}$ Universidade Estadual do Piauí, (myalamonteiro@hotmail.com) \\ ${ }^{7}$ Universidade Estadual do Piauí, (marianacarvalho0173@gmail.com) \\ ${ }^{8}$ Universidade Estadual do Piauí, (ravenamayra@gmail.com) \\ ${ }^{9}$ Universidade Estadual do Piauí, (jorgyannegonzalez1402@gmail.com) \\ ${ }^{10}$ Universidade Estadual do Piauí, (laianasepulveda@hotmail.com)
}

\section{Resumo}

OBJETIVO: Portanto, o objetivo desse estudo é verificar a eficácia dos exercícios na dor em indivíduos com osteoartrite de joelho. MÉTODO: Trata-se de uma revisão sistemática com metanálise, onde foram analisados os estudos publicados entre 2015 a 2021, consultadas nas bases de dados Pubmed, Biblioteca Virtual em Saúde (BVS), Physiotherapy Evidence Database (PEDro). Apenas ensaios clínicos randomizados (ECR) que tivessem recrurado pacientes com diagnóstico de osteoartrite de joelho e estimassem os efeitos de qualquer tipo de exercícios 
comparando com grupo controle e outras intervenções para o desfecho de dor. Oito estudos foram incluídos na análise qualitativa e 7 na metanálise. RESULTADOS E DISCUSSÃO: Mazloum et al (2017) e outros três estudos observaram uma diferença estatística a favor dos exercícios, quando comparados ao grupo controle. E ao ser associado a outras intervenções, seus efeitos foram potencializados, como mostrado por BOKAEIAN et al., (2015) e outros dois estudos. CONCLUSÃO: Pode-se concluir que os exercícios foram eficazes para paciente com dor no joelho devido a osteoartrite e seu efeito foi potencializado quando associado com outras intervenções.

Palavras-chave: Dor; Osteoartrite; Joelho.

Área Temática: Ciências da Saúde

E-mail do autor principal: liliancostaa_@hotmail.com

\section{INTRODUÇÃO}

A osteoartrite, também conhecida como osteoartrose, artrose ou doença articular degenerativa, é uma doença reumática caracterizada pelo desgaste da cartilagem articular e por alterações ósseas e tem como principais sinais e sintomas dor, rigidez matinal, crepitação óssea, atrofia muscular, estreitamento de espaço intra-articular, que resulta em formações osteofíticas, dentre outras manifestações que levam a um declínio funcional. (SOCIEDADE BRASILEIRA DE REUMATOLOGIA, 2019); (YAMADA et al., 2018).

Sua etiologia é multifatorial, mas existem fatores que podem aumentar sua chance de incidência, como a obesidade, o envelhecimento, lesões articulares prévias ou fatores genéticos, por exemplo. Estudos também apontam que a doença tem preferência pela articulação do joelho, devido à sua funcionalidade e características de sustentação e descarga de peso. Além disso, a doença atinge principalmente o sexo feminino (ROCHA, et al., 2020).

As intervenções para a osteoartrite são diversas e aplicadas de acordo com o quadro clínico do paciente e evolução da doença. Entre esses tratamentos, tem ganhado destaque os exercícios, sejam eles em solo ou aquáticos, pois têm demonstrado sucesso quanto à diminuição da dor e melhora da qualidade de vida, uma vez que auxiliam no fortalecimento muscular, propriocepção, nutrição da articulação, dentre outros benefícios que influenciam na manutenção da funcionalidade dos pacientes. (COSTA, 2021); (FACCI; MARQUETTI; COELHO, 2017).

Portanto, o objetivo desse estudo é verificar a eficácia dos exercícios na dor em indivíduos com osteoartrite de joelho. 


\section{MATERIAIS E MÉTODOS}

Trata-se de uma revisão de literatura do tipo sistemática com metanálise.

\subsection{Identificação e seleção de estudos}

Três bases/bancos de dados foram consultadas para elegibilidade dos estudos incluídos, sem restrição de linguagem, com data de publicação entre janeiro de 2016 a março de 2021: Pubmed, Biblioteca Virtual em Saúde (BVS), Physiotherapy Evidence Database (PEDro). Utilizando-se a estratégia de busca a seguir: (movement techniques exercise OR Strength training OR Proprioception training) AND (Knee Osteoarthritis) AND (Pain).

Dois avaliadores independentes analisaram os artigos para elegibilidade (MY e MM) pelo software Rayann QCRI e, em caso de desacordo, um tereceiro revisor arbitrava (LC).

\subsubsection{Delineamento de estudo}

Foram incluídos apenas ensaios clínicos randomizados (ECR).

\subsubsection{Tipos de participantes}

O critério de inclusão dos participantes para inclusão dos estudos foram indivíduos maiores de 18 anos com diagnóstico de osteoartrite de joelho.

\subsubsection{Tipos de intervenções}

Os ensaios foram incluídos apenas se estimassem os efeitos de qualquer tipo de exercícios:

- Exercícios + outra intervenção versus a mesma intervenção;

- Exercícios + outra intervenção versus exercícios;

- Exercícios versus eletroterapia;

- Exercícios versus terapia manual;

- Exercícios versus controle (intervenção mínima, placebo ou nenhuma intervenção).

\subsubsection{Desfecho de interesse}

Dor

\subsection{Avaliação das características dos estudos}


Os revisores extraíram os seguintes dados dos ensaios incluídos: dados bibliométricos, tamanho da amostra, características dos participantes e detalhes das intervenções, medida de desfecho e resultados.

\subsubsection{Risco de viés}

O risco de viés nos ensaios incluídos foi classificado usando a escala PEDro, que também avalia a integridade dos relatórios estatísticos. A pontuação total da PEDro varia de 0 a 10, calculada pela soma do número de critérios alcançados, com exceção do critério 1 , que não é considerado na pontuação final. Os revisores avaliaram qualquer ensaio incluído com a escala PEDro, a menos que uma pontuação fosse já fornecido na página do PEDro (então esta pontuação foi selecionada) (SHIWA et al., 2013). Os ensaios seriam considerados como de "baixo risco" de viés se tivessem uma pontuação maior ou igual a 6 pontos. Ensaios com pontuação menor que 6 foram considerados como tendo "alto risco" de viés (MAHER et al., 2003).

\subsection{Análise dos dados}

Os dados dos resultados foram agrupados e analisados com o Review Manager (RevMan) versão 5.4 (The Nordic Cochrane Center, the Cochrane Collaboration, Copenhagen, Denmark). Com dados contínuos, os resultados foram apresentados como standardized mean difference (diferença das médias padronizadas) com intervalo de confiança de $95 \%$ e modelo de efeito aleatório.

\section{RESULTADOS}

\subsection{Fluxo de estudos através da revisão}

A pesquisa inicial nos bancos de dados eletrônicos identificou um total de 115 registros, após a remoção de duplicadas, foram obtidos 91 estudos e 24 pareciam potencialmente elegíveis após a triagem por título e resumo. A avaliação do texto completo identificou 8 estudos elegíveis, destes 7 foram incluídos na metanálise. O fluxograma do processo da seleção completa e a inclusão de ensaios são apresentados na Figura 01.

\subsection{Características dos estudos incluídos}

\subsubsection{Risco de viés}

E - book Tripé do Ensino Superior: Ensino, Pesquisa e Extensão 
Os resultados da avaliação de risco de viés são apresentados na Tabela 01. A pontuação PEDro dos ensaios incluídos variou de 4 a 7 pontos. Três estudos foram considerados como tendo alto risco de viés e seis com baixo risco de viés.

FIGURA 01: FLUXO DE SELEÇÃO DE ARTIGOS

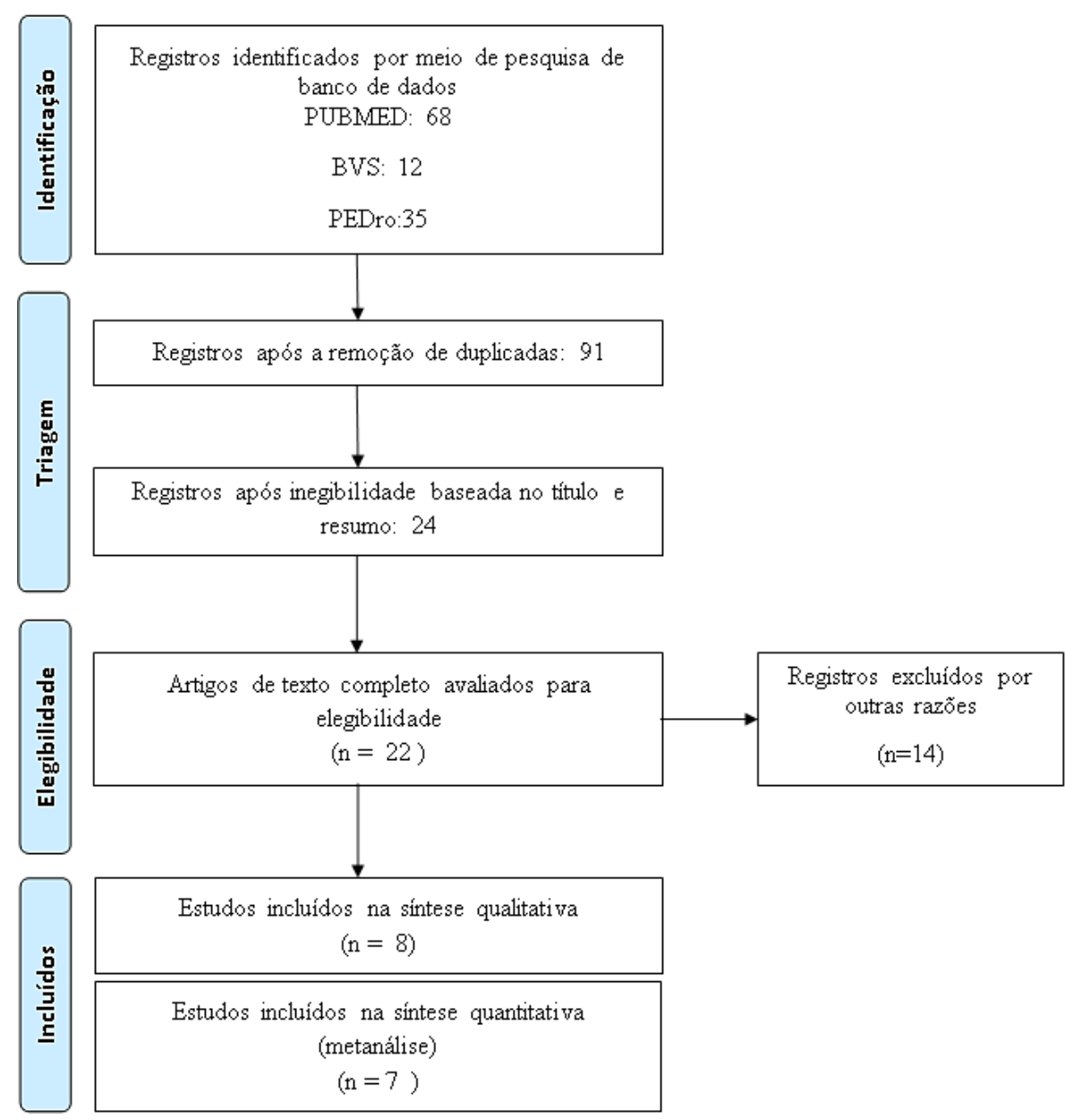

Fonte: Autores, 2021.

TABELA 01: PONTUAÇÃO PEDRO DOS ENSAIOS INCLUÍDOS

\begin{tabular}{|c|c|c|c|c|c|c|c|c|c|c|c|c|}
\hline Estudo & $1^{\circ}$ & $2^{\circ}$ & $3^{\circ}$ & $4^{\circ}$ & $5^{\circ}$ & $6^{\circ}$ & $7^{\circ}$ & $\mathbf{8}^{\circ}$ & $9^{\circ}$ & $10^{\circ}$ & $11^{\circ}$ & Total \\
\hline BOKAEIAN et al., 2015 & $\mathrm{Y}$ & $\mathrm{Y}$ & $\mathrm{Y}$ & $\mathrm{Y}$ & $\mathrm{X}$ & $\mathrm{X}$ & $\mathrm{Y}$ & $\mathrm{Y}$ & $\mathrm{X}$ & $\mathrm{Y}$ & $\mathrm{Y}$ & 7 \\
\hline WANG et al., 2015 & $\mathrm{Y}$ & $\mathrm{Y}$ & $\mathrm{Y}$ & $\mathrm{Y}$ & $\mathrm{X}$ & $\mathrm{X}$ & $\mathrm{Y}$ & $\mathrm{Y}$ & $\mathrm{X}$ & $\mathrm{Y}$ & $\mathrm{Y}$ & 7 \\
\hline MAZLOUM et al., 2017 & $\mathrm{X}$ & $\mathrm{Y}$ & $\mathrm{X}$ & $\mathrm{Y}$ & $\mathrm{X}$ & $\mathrm{X}$ & $\mathrm{Y}$ & $\mathrm{X}$ & $\mathrm{X}$ & $\mathrm{Y}$ & $\mathrm{Y}$ & 5 \\
\hline HALL et al., 2018 & $\mathrm{Y}$ & $\mathrm{Y}$ & $\mathrm{X}$ & $\mathrm{Y}$ & $\mathrm{X}$ & $\mathrm{X}$ & $\mathrm{X}$ & $\mathrm{X}$ & $\mathrm{X}$ & $\mathrm{Y}$ & $\mathrm{Y}$ & 4 \\
\hline HAPER et al., 2019 & $\mathrm{Y}$ & $\mathrm{Y}$ & $\mathrm{X}$ & $\mathrm{Y}$ & $\mathrm{X}$ & $\mathrm{X}$ & $\mathrm{Y}$ & $\mathrm{X}$ & $\mathrm{Y}$ & $\mathrm{Y}$ & $\mathrm{Y}$ & 6 \\
\hline ASSAR ET AL., 2020 & $\mathrm{Y}$ & $\mathrm{Y}$ & $\mathrm{Y}$ & $\mathrm{Y}$ & $X$ & $\mathrm{X}$ & $\mathrm{Y}$ & $X$ & $\mathrm{X}$ & $\mathrm{Y}$ & $\mathrm{Y}$ & 7 \\
\hline YE ET AL., 2020 & $\mathrm{Y}$ & $\mathrm{Y}$ & $\mathrm{X}$ & $\mathrm{Y}$ & $\mathrm{X}$ & $\mathrm{X}$ & $\mathrm{Y}$ & $\mathrm{Y}$ & $\mathrm{Y}$ & $\mathrm{Y}$ & $\mathrm{Y}$ & 7 \\
\hline
\end{tabular}


VINCENT, K.R.;

$\begin{array}{lllllllllll}Y & Y & Y & Y & X & X & Y & X & Y & Y & Y\end{array}$

7

VINCENT, H.K. (2020)

Legenda: $1^{\circ}$ Elegibilidade e fonte $/ 2^{\circ}$ Randomização Aleatória $/ 3^{\circ}$ Sigilo de alocação $/ 4^{\circ}$ Grupos semelhante na linha de base $/ 5^{\circ}$ Cegamento dos participantes $/ 6^{\circ}$ Cegamento terapeuta $/ 7^{\circ}$ Cegamento avaliador $/ 8^{\circ} 15 \%$ de abandonos/ $9^{\circ}$ Análise por intenção de tratar $/ 10^{\circ}$ Diferença relatada entre grupos $/ 11^{\circ}$ Estimativa de ponto e variabilidade relatada/ Y-Sim/ X- Não.

\subsection{Intervenções}

A descrição detalhada das características dos ensaios pode ser encontrada na Tabela 02.

TABELA 02: CARACTERÍSTICAS DOS ENSAIOS

\begin{tabular}{|c|c|c|c|}
\hline Estudo & Participantes & Intervenção & Medidas de desfecho \\
\hline $\begin{array}{l}\text { Bokaeian } \\
\text { et al., } 2015\end{array}$ & $\mathrm{~N}: 28$ & $\begin{array}{l}\text { G1: treino de força } \\
\text { G2: treino de força } \\
+\quad \text { vibração de } \\
\text { corpo inteiro }\end{array}$ & 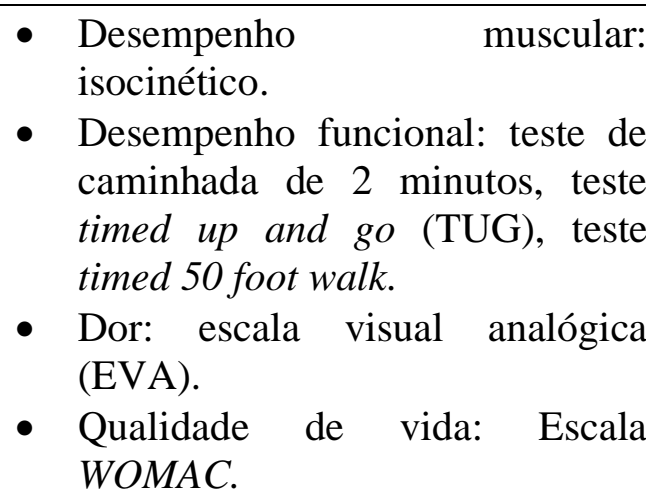 \\
\hline
\end{tabular}

$\begin{array}{lrl}\text { Wang et N: } 99 & \text { G1: treino de } \\ \text { al., 2015 } & \text { resistência } \\ & \text { G2: treino de } \\ & \text { resistência }+ \\ & \text { vibração de corpo } \\ & \text { inteiro }\end{array}$

Mazloum N: $31 \quad$ G1: exercícios de et al., 2017

Hall et al., N: $97 \quad$ G1: exercícios de 2018
Pilates

G2: exercícios terapêuticos

G3: nenhuma intervenção

fortalecimento para extensores de joelho
- Função física e desempenho funcional: TUG, teste de caminhada de 6 minutos, escala WOMAC.

- Dor: EVA.

- Força muscular: dinamômetro hand-held.

- Atividade da vida diária: índice de Lasegue.

- Qualidade de vida: questionário SF-36.

- Incapacidade e dor: índice de Lequesne.

- Desempenho funcional: tempo necessário para realizar atividades de caminhar, sentar e levantar.

- Senso de posição articular: Biodex.

- Função física e dor: escala WOMAC.

- Força muscular: dinamômetro isocinético.

\section{E - book Tripé do Ensino Superior: Ensino, Pesquisa e Extensão}




\begin{tabular}{|c|c|c|c|}
\hline & & $\begin{array}{l}\text { G2: nenhuma } \\
\text { intervenção }\end{array}$ & \\
\hline $\begin{array}{l}\text { Haper et } \\
\text { al., } 2019\end{array}$ & $\mathrm{~N}: 35$ & $\begin{array}{l}\text { G1: exercícios de } \\
\text { resistência } \\
\text { G2: exercícios de } \\
\text { resistência }+ \\
\text { compressão } \\
\text { isquêmica }\end{array}$ & $\begin{array}{l}\text { - Força muscular: dinamômetro } \\
\text { Biodex. } \\
\text { - Velocidade de caminhada: andar } \\
10 \text { voltas em um percurso de } 40 \\
\text { metros. } \\
\text { - Dor: EVA e Escala WOMAC. } \\
\text { - Função física: Short Physical } \\
\text { Performance Battery e Late Life } \\
\text { Function and Disability } \\
\text { Instrument. }\end{array}$ \\
\hline $\begin{array}{l}\text { Assar et } \\
\text { al., } 2020\end{array}$ & $\mathrm{~N}: 36$ & $\begin{array}{l}\text { G1: programa de } \\
\text { exercícios } \\
\text { baseado em TRX } \\
\text { G2: programa de } \\
\text { exercícios } \\
\text { aquáticos } \\
\text { G3: nenhuma } \\
\text { intervenção }\end{array}$ & $\begin{array}{l}\text { - } \text { Dor: VAS. } \\
\text { - Rigidez da articulação: WOMAC. } \\
\text { Equilíbrio: Escala de equilíbrio de } \\
\text { Berg. } \\
\text { - Instabilidade do joelho: } \\
\text { questionário de Felson, escala } \\
\text { numérica (0-5). } \\
\text { - Amplitude de movimento de } \\
\text { flexão de joelho: inclinômetro de } \\
\text { bolha. } \\
\text { - Força isométrica máxima dos } \\
\text { extensores do joelho: Baseline } \\
\text { Pull Push Dynamometer. }\end{array}$ \\
\hline $\begin{array}{l}\text { Ye et al., } \\
2020\end{array}$ & $\mathrm{~N}: 50$ & $\begin{array}{l}\text { G1: Baduanjin } \\
\text { Qigong } \\
\text { G2: nenhuma } \\
\text { intervenção }\end{array}$ & $\begin{array}{l}\text { - Estabilidade postural: sistema } \\
\text { Prokin. } \\
\text { - Posição do centro de pressão: } \\
\text { deslocamento anterior posterior } \\
\text { (AP) e médio-lateral (ML) } \\
\text { medidos em milímetros. } \\
\text { - Estabilidade postural } \\
\text { propriocepção no joelho: Sistema } \\
\text { Proki. } \\
\text { - Nível de dor, rigidez articular e } \\
\text { função física: WOMAC. }\end{array}$ \\
\hline $\begin{array}{l}\text { Vincent, } \\
\text { K.R.; } \\
\text { Vincent, } \\
\text { H.K. } \\
(2020)\end{array}$ & $\mathrm{N}: 53$ & $\begin{array}{l}\text { G1: treinamento } \\
\text { de resistência } \\
\text { concêntrica } \\
\text { G2: treinamento } \\
\text { de resistência } \\
\text { excêntrico } \\
\text { G3: nenhuma } \\
\text { intervenção }\end{array}$ & $\begin{array}{l}\text { - Dor: escala Numérica de Dor de } \\
11 \text { pontos } \\
\text { - Função física: teste de elevação da } \\
\text { cadeira, teste de subida de escada, } \\
\text { teste de caminhada e teste de } \\
\text { caminhada de } 6 \text { minutos } \\
\text { - Força: método de } 1 \mathrm{RM}\end{array}$ \\
\hline
\end{tabular}




\subsection{Efeitos dos tratamentos}

\subsubsection{Comparação entre exercício $X$ controle}

Quatro estudos (MAZLOUM et al., 2017; HALL et al., 2018; ASSAR et al., 2020; YE et al., 2020) compararam exercícios com grupo controle (intervenção mínima, placebo ou nenhuma intervenção) e verifica-se que há diferença estatística entre os grupos a favor dos exercícios (SMD= -0,70; IC 95\% -1,25 a -0.31; p=002) (Figura 02).

\section{FIGURA 02: METANÁLISE EXERCÍCIO X CONTROLE}

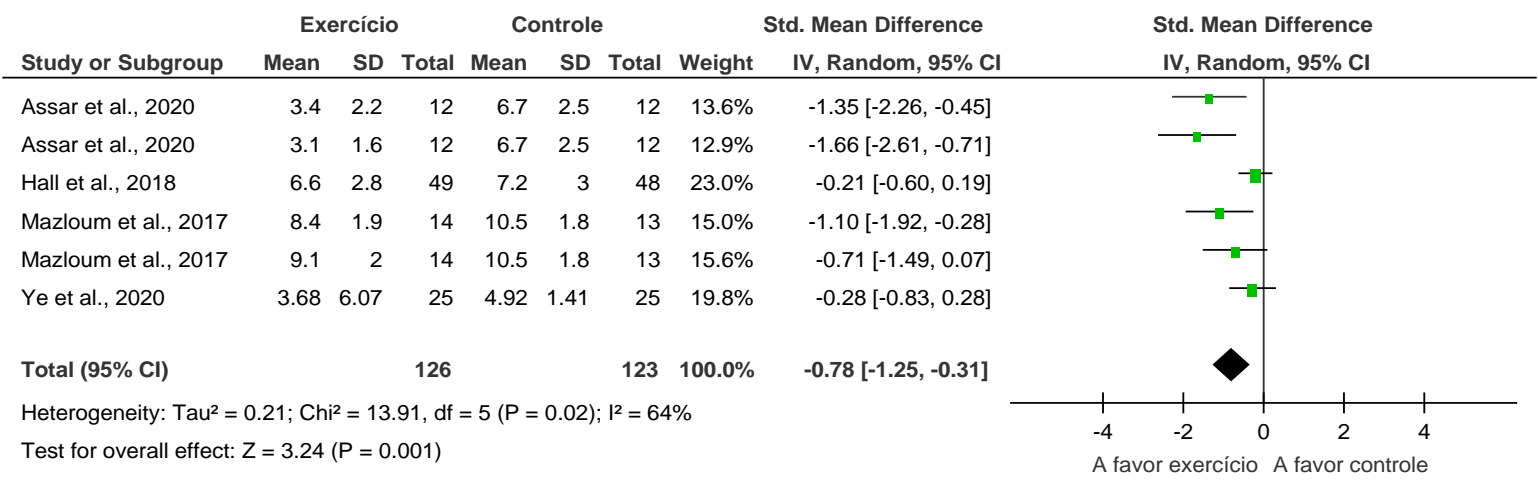

Legenda: Forest plot para comparação: 1 Exercício X controle, desfecho: 1.1 Dor.

\subsection{Comparação entre exercício $X$ exercício + outra intervenção}

Três estudos (BOKAEIAN et al., 2015; WANG et al., 2015; HAPER et al., 2019 ) compararam exercícios com exercícios + outra intervenção e verificou-se que há diferença estatística entre os grupos a favor dos exercícios + outra intervenção (SMD= 0,73; IC 95\% 0.16 a $1.30 ; p=0.08)($ Figura 03).

FIGURA 03: METANÁLISE EXERCÍCIO X EXERCÍCIO + OUTRA INTERVENÇÃO

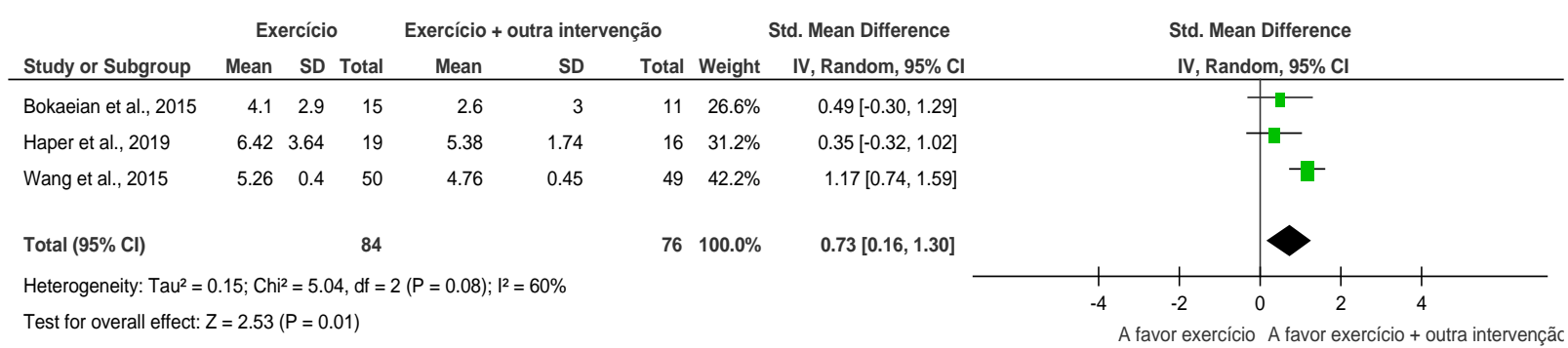

Legenda: Forest plot para comparação: 2 Exercício X exercício + outra intervenção, desfecho: 2.1 Dor.

\section{E - book Tripé do Ensino Superior: Ensino,} Pesquisa e Extensão 


\section{CONSIDERAÇÕES FINAIS}

Os exercícios foram mais eficazes dos que grupos controles e seu efeito foi potencializado quando associado com outras intervenções. Assim, recomenda-se o seu uso para paciente com dor no joelho devido à osteoartrite.

\section{REFERÊNCIAS}

ASSAR, S. et al. The effect of Total resistance exercise vs. aquatic training on self-reported knee instability, pain, and stiffness in women with knee osteoarthritis: a randomized controlled trial. BMC Sports Science, Medicine and Rehabilitation, v. 12, n. 1, p. 1-13, 2020 .

BOKAEIAN, H.R. et al. The effect of adding whole body vibration training to strengthening training in the treatment of knee osteoarthritis: A randomized clinical trial. Journal of bodywork and movement therapies, v. 20, n. 2, p. 334-340, 2016.

CHEN, H. et al. The effects of a home-based exercise intervention on elderly patients with knee osteoarthritis: a quasi-experimental study. BMC musculoskeletal disorders, v. 20, n. 1, p. 1-11, 2019.

COSTA, M.S. Recursos fisioterapêuticos utilizados na osteoartrite de joelho, 2021.

FACCI, L.M.; MARQUETTI, R.; COELHO, K. C. Fisioterapia aquática no tratamento da osteoartrite de joelho: série de casos. Fisioterapia em movimento, v. 20, n. 1, 2017.

HALL, M. et al. Knee extensor strength gains mediate symptom improvement in knee osteoarthritis: secondary analysis of a randomised controlled trial. Osteoarthritis and cartilage, v. 26, n. 4, p. 495-500, 2018.

HARPER, S.A. et al. Blood-flow restriction resistance exercise for older adults with knee osteoarthritis: a pilot randomized clinical trial. Journal of clinical medicine, v. 8, n. 2, p. $265,2019$.

MAHER, C.G. et al. Reliability of the PEDro scale for rating quality of randomized controlled trials. Phys Ther, v. 83, n.8, p.713-21, 2003.

MAZLOUM, V. et al. The comparison of the effectiveness of conventional therapeutic exercises and Pilates on pain and function in patients with knee osteoarthritis.

Complementary therapies in clinical practice, v. 31, p. 343-348, 2018.

OUYANG, X. et al. The curative efficacy of arthroscopic therapy in treating anterior cruciate ligament rupture with secondary osteoarthritis. Eur Rev Med Pharmacol Sci, v. 20, n. 2, p. 214-219, 2016. 
ROCHA, T.C. et al. Os efeitos do exercício físico sobre o manejo da dor em pacientes com osteoartrose de joelho: Uma revisão sistemática com meta-análise. Revista Brasileira de Ortopedia, v. 55, n. 5, p. 509-517, 2020.

SARDIM, A.C.; PRADO, R.P.; PINFILDI, C.E. Efeito da fotobiomodulação associada a exercícios na dor e na funcionalidade de pacientes com osteoartrite de joelho: estudo-piloto. Fisioterapia e Pesquisa, v. 27, p. 119-125, 2020.

SHIWA, S.R. et al. Language of publication has a small influence on the quality of reports of controlled trials of physiotherapy interventions. J Clin Epidemiol, v.66, n.1, p.78-84, 2013. SOCIEDADE BRASILEIRA DE REUMATOLOGIA - SBR. Osteoartrite (Artrose), 2019. Disponível em: <https://www.reumatologia.org.br/doencas-reumaticas/osteoartrite-artrose/>. Acesso em: 13 set 2021.

VINCENT, K.R.; VINCENT, H.K. Concentric and Eccentric Resistance Training Comparison on Physical Function and Functional Pain Outcomes in Knee Osteoarthritis: A Randomized Controlled Trial. American journal of physical medicine \& rehabilitation, v. 99, n. 10, p. 932-940, 2020.

WANG, P. et al. Effects of whole body vibration exercise associated with quadriceps resistance exercise on functioning and quality of life in patients with knee osteoarthritis: a randomized controlled trial. Clinical rehabilitation, v. 30, n. 11, p. 1074-1087, 2016.

YAMADA, E.F. et al. Efeito dos exercícios de fortalecimento, de marcha e de equilíbrio no tratamento de osteoartrite de joelho. Revista Brasileira de Ciência e Movimento, v. 26, n. 3, p. 5-13, 2018.

YE, J. et al. The effects of baduanjin qigong on postural stability, proprioception, and symptoms of patients with knee osteoarthritis: a randomized controlled trial. Frontiers in medicine, v. 6, p. 307, 2020. 